\title{
4 \\ Endemic Mycoses and Allergies: Diseases of Social Change
}

In 1950, the Biology Section of the New York Academy of Science (NYAS) held what it claimed to be the first conference on medical mycology in the United States. ${ }^{1}$ What prompted the event was not the announcement of the discovery of nystatin by Hazen and Brown, as their publication was still in press, but the growing profile of fungi and fungal infections across the nation. Fungi, not least because of interest in penicillin, were attracting the interest of biologists and biomedical researchers who, alongside screens for antibiotic activity, were adopting them as experimental models in studies of nutrition, physiology and immunology. ${ }^{2}$ All the leading names of the field from the 1930s attended the meeting: Carroll Dodge, Norman Conant, Rhoda Benham and Lucille Georg, and there were new faces who had developed expertise during the war and in particular localities. Speakers drew attention to the increased incidence of systemic candidiasis, signalling a switch in the medical mycological gaze from external (exogenous) to internal (endogenous) disease. Although the incidence of endogenous, systemic fungal infections was very low, they had very high mortality and presented unusual cases that fascinated physicians. In addition, there was a new awareness of the toll of morbidity from endemic, exogenous disease, as with athlete's foot and thrush, and with regionally specific, often sub-clinical infections, principally coccidioidomycosis, blastomycosis and histoplasmosis.

In this chapter, we tell the story of regionally specific fungal infections, and look at the rise of fungal-induced asthma, as one part of the twentieth-century story of the rise of allergies and asthma. ${ }^{3}$ We begin by discussing the new epidemiology of endemic fungal infections that emerged in the late 1940 s and the attempts by medical mycologists and other interested clinicians to attract more resources for research, 
prevention and control. We discussed the increased incidence of acute, invasive candidiasis associated with new medical treatments in the last Chapter; here we examine in turn the three principal chronic, though occasionally epidemic, regional mycoses prevalent in North America coccidioidomycosis, blastomycosis and histoplasmosis. Our attention then switches to Britain and allergic fungal conditions, firstly, farmer's lung and then allergic bronchopulmonary aspergillosis (ABPA). This class of fungal allergens was 'discovered' in Britain and seemingly absent from North America, until expertise was transferred back across the Atlantic.

\section{The new epidemiology of fungal diseases}

In the early 1950s, medical mycologists, along with cancer physicians and chest surgeons, began to draw attention to a new problem posed by invasive fungal infections. Writing in 1953, David Smith, a colleague of Norman Conant at Duke Medical School described the new situation as follows.

Unlike most bacterial and viral infectious diseases, the systemic mycotic infections are not transmitted directly from patient to patient; consequently, one would not expect to see epidemics caused by fungi. In most instances the mycotic infections are endemic and sporadic but true epidemics of sporotrichosis, coccidioidomycosis and histoplasmosis do occur when groups of non-immune individuals are exposed to an environment containing large amounts of the saprophytic form of the fungus. More than a thousand cases of sporotrichosis developed in the gold mines of South Africa when the timbers in the mine became infected with Sporotrichum schenckii. Epidemics of coccidioidomycosis occurred when companies of nonimmune soldiers from the East marched in the dust of certain Southwestern deserts. Epidemics of histoplasmosis have occurred following the exposure of groups of nonimmune individuals to pigeon manure, chicken manure, bat manure in caves and to the dust of unused silos. ${ }^{4}$

Reviewing the epidemiology of fungal infections in the same year for the New England Journal of Medicine, Otis Jillson pointed to 'the recognition of the benign, common forms of histoplasmosis; the diagnosis and surgical treatment of coccidioidal pulmonary residua; the treatment of blastomycosis with stilbenes', and added the growing incidence of systemic mycoses. ${ }^{5}$ Interestingly, he dealt with skin infections briefly 
and said very little about exogenous or endogenous candidiasis, at a time when nystatin was attracting attention.

At the 1950 NYAS meeting, Samuel B. Salvin, then at the Division of Infectious Diseases at NIH, placed the incidence of fungal diseases in context of other infectious diseases.

Fungus infections in man, although less frequent than bacterial, are still numerically important. For example, of the 92,933 deaths due to infections and parasitic diseases in the United States in 1945, 284, or 0.3 per cent, were due to mycoses. This was approximately equal to the number of deaths reported as caused by scarlet fever, measles, or the typhus-like diseases (due to rickettsia), and was more than the total of all deaths recorded from rabies, smallpox, relapsing fever, leprosy, brucellosis, paratyphoid fever, plague, cholera, and anthrax. It should be realized, of course, that effective control measures are employed against some of the aforementioned diseases, whereas control methods against the mycoses not only are not practiced, but, generally, are not even known. It should also be borne in mind that the dermatophytoses, although characteristically nonfatal, are extremely common, probably equalling the most widespread of the bacterial or virus diseases in prevalence. ${ }^{6}$

In 1953, Walter Nickerson from the Department of Microbiology, Rutgers University, tried a creative presentation of mortality data to chart the rise of fungal infections. ${ }^{7}$ He used graphs for the period 1945-1949 that showed starkly opposite mortality trends, where 'all infections' had dropped sharply, while that from mycoses had increased markedly. Nickerson had produced his graphic illustration by using very different scales for the two classes of infection. Deaths from mycoses were recorded as actual numbers, while those from all infections were recorded in thousands. The alarming increase of nearly $50 \%$ in fungal infection deaths was actually from 270 to 380, while the actual number of deaths from all infections had fallen 30\%, from 93,000 to 66,000 deaths - still 170 times greater! In the event, the annual total deaths from mycoses in the next decade never reached $500 .{ }^{8}$ More telling was Nickerson's point about morbidity; he stated that 'mycotic infections are probably the mostly widely distributed and most numerous types of infection, with dermatophyte infections, such as athlete's foot, alone as prevalent as the most widespread of the bacterial and virus diseases'. ${ }^{9}$

Medical mycologists had long argued for recognition of the distinctive pathogenicity of fungi. In 1940, Arthur Henrici, who worked in the Department of Bacteriology at the University of Minnesota, argued 
that most bacterial and viral infections developed rapidly, and then plateaued in severity, before falling away, because of either recovery or death. With fungal infections, however, a typical pattern was of the slow and incremental development of chronicity, and prolonged morbidity, often at sub-clinical levels. ${ }^{10}$ The only bacterial diseases with similar patterns of pathogenicity were tuberculosis and leprosy. Henrici maintained that the slow increase in severity meant either that the infecting fungus progressively changed and developed pathogenic properties or that the resistance of the host was gradually worn down. There was no evidence of the former, so he focused on changes in the host and its 'soil', claiming that prolonged exposure to certain fungi and their toxins produced hypersensitivity in the host cells. Henrici referred back to the theories of Richard Pfeiffer and Clemens von Pirquet on overactive immune responses. ${ }^{11}$ Pfeiffer's authority was drawn upon for the argument that fungal endotoxins inflamed and ultimately killed cells, creating a nidus for the fungus itself to grow. On the other hand, von Pirquet's work was used to suggest that host cells developed allergic-type sensitivity. Henrici favoured the latter, but stressed that because fungal infections were complex and variable, both mechanisms might operate, or be found with different species of pathogen.

The prevalence of sub-clinical, chronic disease was recognised by public health officials as characteristic of regionally specific mycoses in the United States. The new geography of endemic fungal infections was revealed by David Smith in 1953, on a map that showed the distribution of coccidioidomycosis, blastomycosis and histoplasmosis in the United States and northern Mexico ${ }^{12}$ (Figure 4.1). The new epidemiological profile changed the position of medical mycology in the United States in the 1950s. This was evident first in the proliferation of new publications and courses. ${ }^{13}$ Ana Espinel-Ingroff's analysis of the institutional development of medical mycology shows that new departments and new experts emerged in affected areas, for example, at Michigan State University (1951), Tulane University (1955), the University of California, Los Angeles (1956), the University of Oklahoma (1957) and Virginia Commonwealth University (1965). ${ }^{14}$ Initiatives took place in varied settings, sometimes with public health departments, but mostly in university biology and microbiology departments. That said, leading figures and departments in the East remained important. Norman Conant, Chester Emmons and Libero Ajello continued to head key departments at Columbia, Duke and the National Microbiological Institute, which was reorganised from 1955 as the National Institute of Allergy and Infectious Diseases (NIAID) and, of course, the Centers for Disease Control and Prevention (CDC) in Atlanta remained influential. 


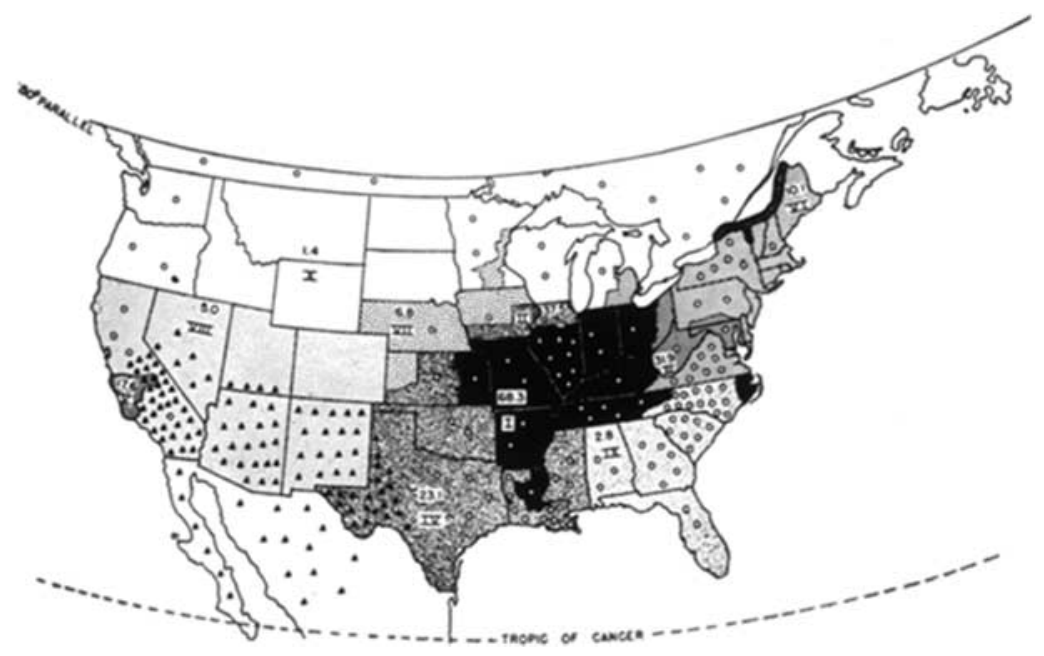

Figure 4.1 Distribution of histoplasmosis, blastomycosis and coccidioidomycosis in North America. ${ }^{15}$ The histoplasmosis areas are shown in black, the blastomycosis as circles and the coccidioidomycosis areas as triangles. Smith, D. T., 'The diagnosis and therapy of mycotic infections', Bull NY Acad Med, 1953, 29(10): 778. This figure (c) 2013 New York Academy of Medicine used under Creative Commons Attribution - Non-commercial licence: http://creativecommons.org/ licenses/by/3.0/

\section{Regional mycoses I: 'Valley fever' - coccidioidomycosis}

The first regionally specific infection to attract attention was coccidioidomycosis through Emmons's work at NIH. ${ }^{16}$ The very notion of the geographically specific infection had weakened in medicine in the second half of the nineteenth century with the move towards aetiological definitions of disease and the essential role of specific germs. However, there was new interest in the early twentieth century with vector-borne tropical diseases, where the range of insects was limited by biogeographical factors. ${ }^{17}$ In the United States this was evident in the work on tick-borne Rocky Mountain Spotted Fever, which had its highest prevalence in certain states: North Carolina, Oklahoma, Arkansas, Tennessee, and Missouri. ${ }^{18}$

In the 1940s, coccidioidomycosis, also styled as 'San Joaquin' or 'Valley fever', was known to be caused by the fungus Coccidioides immitis (C. immitis) ${ }^{19}$ Its mode of spread was seemingly simple: fungal spores, released from the soil, entered the body through the lungs, where an infection might develop. In fact, most people did not develop any 
symptoms and those that did experienced a cold or mild flu-like symptoms. In a tiny fraction of people, especially those with other diseases and with weakened immune systems, infection spread through the bloodstream to give disseminated coccidioidomycosis.

Research by Ernest C. Dickson and Charles Smith in the 1930s had revealed that infection with $C$. immitis was very common amongst those living in certain areas, and that it was best regarded as an endemic, chronic and benign infection..$^{20}$ It seemed that residents built up immunity from long-term, low-level exposure, hence most infection was sub-clinical and, as would be expected, clinical disease was most common amongst in-migrants who had not had the opportunity to build up immunity. Population movement and settlement westwards had been going on in the United States for many decades and it seemed likely that coccidioidomycosis emerged at this particularly moment because of the new speed and scale of migration, especially families fleeing dust blows in the prairies. Evidence to support this view came in the 1940s when coccidioidomycosis developed amongst the recruits brought to the region to train for the United States Air Force. ${ }^{21}$ As the war effort grew, more troops arrived, which gave investigators the opportunity to make comparisons of incidence by sex, race and nationality. For instance, some 13,000 German prisoners of war were held at Florence, Arizona, where the incidence of coccidioidomycosis became so high that they were moved away, as United States government officials worried that such a high rate of infection would lead to them being charged with violation of the Geneva Convention on treatment of war prisoners. ${ }^{22}$

After the war, the military presence in the Southwest continued and expanded. So too did worries about coccidioidomycosis and this led in 1955 to the establishment of an annual meeting of the Veterans Administration-Armed Forces Coccidioidomycosis Cooperative Study Group (CCSG). This type of cooperative meeting had begun in the late 1940s as a way of developing and sharing expertise on the treatment of tuberculosis with streptomycin. ${ }^{23}$ Initially, the main agencies were the Veterans Administration, the Army, the Navy, Public Health Services and the National Research Council. While the focus of the early annual conferences was squarely on tuberculosis, fungal infections were sometimes discussed, as in 1952 when trial coordinators noted that histoplasmosis lung infections had complicated their clinical trials by making differential diagnosis with X-rays more difficult. ${ }^{24}$ The first coccidioidomycosis meeting in 1955 came from a direct concern with the growing incidence of the infection, especially at air bases, notably Williams AFB 
and Luke AFB in Arizona, Edwards AFB in California, and Lemoore NAS, also in California. The problem grew with the investments in military infrastructure that came with the Cold War. Williams and Luke became training centres, with a steady stream of non-immune recruits passing through, while Lemoore was further developed in the 1960s for strike aircraft, and Edwards became a centre for research, including rocketry, and eventually was a landing site for the Space Shuttle.

The construction of new runways and other facilities on these military sites disturbed the subsoil, which together with aircraft take-offs and landings circulated C. immitus spores to those living and working nearby. ${ }^{25}$ Some civilian sufferers were treated by military doctors; however, they were mostly dealt with by local physicians, who also treated military personnel when they were referred to local hospitals for serological and radiological investigation. Thus, the leading authority on the disease in the military, from the 1940s to the 1960s, was Charles E. Smith, Dean of Public Health at the University of California Berkeley. ${ }^{26}$ He had developed his expertise during the Second World War at Berkeley, where he established a research laboratory and diagnostic serological services.

In the 1950s C. immitus biology was found to be more complicated than previously recognised and the new understanding was set out in the first book entirely on the disease by Marshall J. Fiese in 1958. ${ }^{27}$ Fiese was based at the Veterans Administration Hospital in Fresno and in his 'Foreword' to Fiese's volume, Charles Smith wrote that the work reflected the author's deep experience: '[Fiese] has seen and viewed .... countless roentgenograms[X rays], seen and studied the tragic autopsies, and perhaps most importantly of all, lived for years in the coccidioidal countryside.' An unusual feature of C. immitis was that, rather than being spread by spores, it was actually the cells of the hyphae, called conidia, that circulated in the air. Conidia were found to be tiny and readily carried in dust; hence, the popular representation of coccidioidomycosis as spread by 'flying conidia', or 'flying chlamydospores'. ${ }^{28}$ When inhaled, the human body was shown to respond in one of three possible ways. First and most commonly, the conidia were destroyed by the immune system and a degree of immunity, albeit variable, to future infection was established. Alternatively, the conidia grew in the lungs in a spherical form, into bodies that released many more such 'spherules', producing inflammation and a chest infection. Lastly and least common, and only if the lung infection was severe, infection could spread in the blood and cause inflammation, especially in the skin and brain. The reported incidence of the severe 
form increased in the 1960s and was found principally amongst patients and former patients who were to some degree immuno-compromised. ${ }^{29}$

Local physicians in Arizona and California had mostly to deal with the second type of endemic infection, which was often self-limiting. ${ }^{30}$ Particular occupational groups were at higher risk of developing the disease, notably, agricultural and construction workers. Archaeologists were another high-risk group, and often had severe infection because they were new to a region. The profile of coccidioidomycosis rose in sporadic epidemic outbreaks, as in California at the end of 1977. A large dust storm blew through Bakersfield on 20 December, depositing conidia to the north and west along familiar terrain in the San Joaquin Valley. ${ }^{31}$ Cases were reported in two main areas: the known endemic area of Kern County around Bakersfield and a previously non-endemic area west of Sacramento. Within six months, 142 cases of clinical lung disease had been identified in Kern County and 379 at the University of California Davis (UCD), nearly 300 miles north, with sufferers from Los Angeles and Oakland, as well as Sacramento. Public health officials reported that rainfall immediately after the storm had probably reduced significantly the number of cases; however, they worried that conidia had drained into the subsoil and that new endemic areas might be created. ${ }^{32}$ Demosthenes Pappagianis, a member of the Department of Medical Microbiology at UCD who had led the local response to the epidemic, became a leading researcher on the epidemiology of the disease in subsequent decades ${ }^{33}$ (Figure 4.2).

In the early 1990s, there was another epidemic, later termed 'the great coccidioidomycosis outbreak'. Reported cases of the infection rose from a long-term average of 300-600, to 1,200 in 1991, to 4,541 in 1992 and 4,107 in $1993 .^{34}$ The foci were in the south of the San Joaquin Valley in Kern and Tulare counties. Pappagianis was brought in to investigate. He found no obvious precipitating reason for the outbreak, and instead looked to climatic factors, notably the long-term drought and high spring rainfall in 1991 and 1992, to soil disturbance from construction; and to possible new groups of susceptible in-migrants. ${ }^{35}$ The outbreak brought national attention to coccidioidomycosis and a CDC-led investigation, whose interest was in both endemicity and the extent of acute and disseminated disease. ${ }^{36}$ Their study concluded that with the aging of the US population and the increase in the number of immunosuppressed persons, severe pulmonary and disseminated coccidioidomycosis threaten to become important public health problems in areas of endemicity. ${ }^{37}$ Pappagianis also contributed to an investigation of an outbreak following an earthquake in Northridge, 


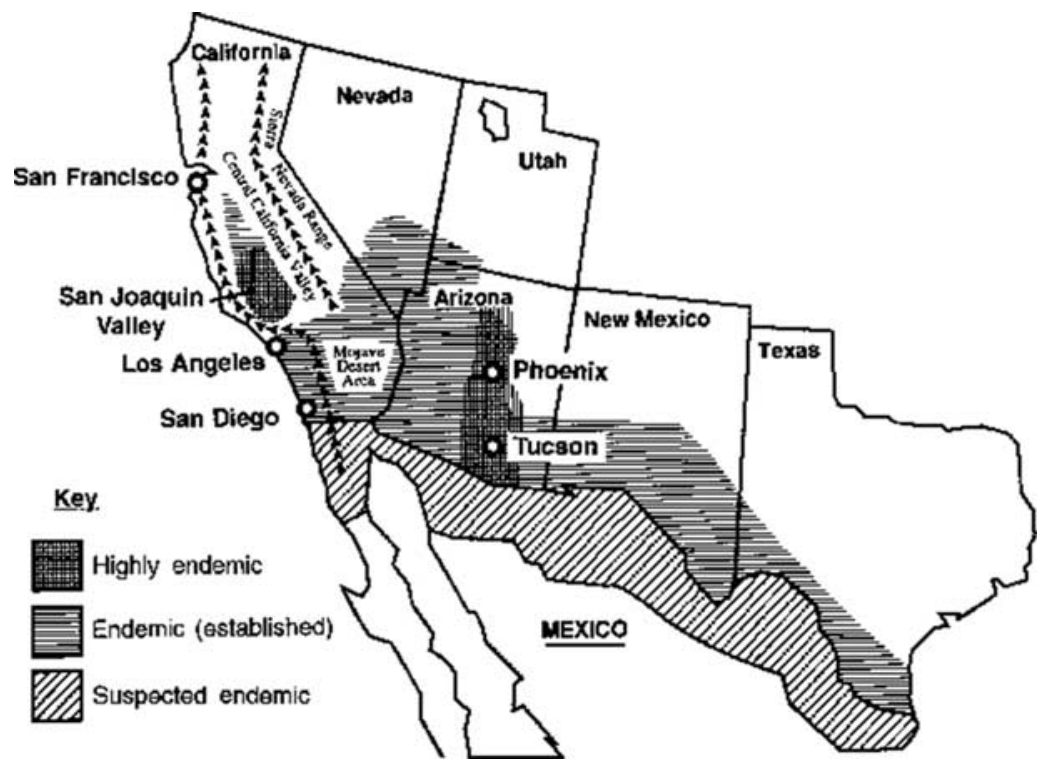

Figure 4.2 The geographic distribution of coccidioidomycosis. Cross-hatching indicates the heavily disease-endemic area, single hatching and the moderately disease-endemic area. ${ }^{38}$ Kirkland, T. N. and Fierer, J., 'Coccidioidomycosis: A reemerging infectious disease', Emerg Infect Dis [serial on the Internet]. 1996, Sep. Available from www.nc.cdc.gov/ncidod/eid/vol2no3/kirkland.htm DOI: 10.3201/eid0203.960305. CDC Public domain material.

California in January 1994, to which 203 cases were linked and three coccidioidomycosis deaths added to the overall toll of $57 .{ }^{39}$

In addition to Pappagianis, two other researchers stood out in the study of coccidioidomycosis during the period. One was John Galgiani, who worked at the Veterans Affairs Medical Center and University of Arizona, Tucson, and was the leading expert on coccidioidomycosis in that state. His specialism was immune responses and he was amongst the first to publish on coccidioidomycosis in AIDS sufferers. ${ }^{40}$ Over many years he campaigned for recognition that coccidioidomycosis was becoming a disease of national importance, because of continuing high rates of in-migration to Southwestern states and the increase in the number of immunosuppressed patients. General population mobility was also a factor, with many cases presenting outside of endemic areas in out-migrants; indeed, he reported that $46 \%$ of coccidioidomycosis patients with AIDS were in non-endemic areas. ${ }^{41}$ In 1996, coccidioidomycosis was being discussed as 'a re-emerging infectious disease'. ${ }^{42}$ 
Theo Kirkland and Joshua Fierer, respectively, from the San Diego School of Medicine and Department of Veterans Affairs Medical Center, San Diego, wrote that it warranted the label because 'the number of cases... has increased dramatically, and the clinical symptoms of this illness have changed in patients with acquired immunodeficiency syndrome (AIDS)' ${ }^{43}$

The second key researcher was David A. Stevens, who was based in the Santa Clara Valley Medical Center, San José and the Stanford University School of Medicine. He became a leading authority on the clinical management of coccidioidomycosis, especially the use of chemotherapy. His expertise, developed along with that on the treatment of aspergillosis and other opportunistic mycoses, was recognised in leadership roles and honours in the Medical Mycological Society of the Americas (MMSA), International Society for Human and Animal Mycology (ISHAM) and the American Society for Microbiology (ASM). Stevens's early career work was on viruses, but he switched to fungi in 1974 with a study of immunity to C. immitis and then to its treatment. ${ }^{44}$ With Hillel B. Levine, he pioneered the use of one of the new azoles, miconazole, for coccidioidomycosis. ${ }^{45}$ In 1980, he edited a new textbook on the disease, contributing chapters on immunology, other syndromes, immuno-compromised hosts, chemotherapy immunotherapy, vaccination and a bibliography. ${ }^{46}$ Stevens enrolled Pappagianis to write on epidemiology and serology, and Galgiani on ophthalmic disease.

By the 1970s, the Cooperative Study Group meetings, which began under military sponsorship, had moved to civilian control, with Stevens, Galgiani and Pappagianis taking leading roles. However, as early as 1957 a larger symposium had been called and that met every eight years or so, styling itself as 'international' for the first time when it met in Tucson in $1977 .{ }^{47}$ This change reflected two developments: first, greater interest in the disease in Mexico and South America and second, the experience of physicians across borders with serological diagnosis and amphotericin B treatment of disseminated disease. ${ }^{48}$ Furthermore, after the Californian coccidioidomycosis outbreak in 1977, a growing number of laboratories had begun to explore vaccine development, utilising the tools of the new molecular biology. ${ }^{49}$ Indeed, a vaccine developed by Pappagianis and Levine went on trial in 1981, but this showed little or no benefit. ${ }^{50}$ Attention was also directed to ethnic and social groups that were at greater risk from the disease, particularly black men, Native Americans, and pregnant women. With the former, researchers explored the contribution of racial susceptibility and environmental factors, with a consensus developing around the importance of the latter. 
Researchers found that exposure, socio-economic position and access to health care were the most important determinants of vulnerability to infection and the development of disease. ${ }^{51}$ Pregnant women were thought to be vulnerable because of changes in their immunity and hormonal levels, but close study showed them to have, at worst, only a tiny additional likelihood of developing disseminated disease. ${ }^{52}$ In the 1980s, as would have been expected, more coccidioidomycosis was reported in immuno-compromised patients in Southwestern states, and also across the United States in people who had lived or travelled to the endemic areas. Thus, the work of regional coccidioidomycosis experts linked up with that of mycologically minded clinicians nationwide. Their research and clinical experience put them in a good position to assume national leadership roles in the field. For example, David Stevens led the NIH multicentre clinical trials group on antifungal drugs between 1990 and 2000 and chaired committees writing practice guidelines on aspergillosis as well as coccidioidomycosis. ${ }^{53}$

\section{Regional mycoses II: Blastomycosis - North American and otherwise}

Blastomycosis was a term invented at the end of the nineteenth century for eruptive skin lesions or granulomas, which were assumed to be caused by infective fungi. The prefix 'blasto-', from the Greek for 'budding' or 'sprouting', came from the clinical presentation of raised lesions that were disfiguring, especially so with facial lesions. In the 1900 s in the United States, the name became associated with so-called Gilchrist's disease, first thought to be protozoan, but then linked to a dimorphic (two forms) fungus named Blastomyces dermatitidis (B. dermatitidis). ${ }^{54}$ For a while the infection was termed 'North American blastomycosis' as researchers found that it was restricted to the geographical areas of the Ohio, Mississippi and Missouri River basins and the western shore of Lake Michigan. There followed, what contemporaries recognised as, 'an era of confusion during which the disease was confounded with other entities, particularly cryptococcosis and candidiasis' and 'Nineteen new names were suggested for the causative fungus. ${ }^{55}$ Consensus on the pathogenesis of the disease followed work by Norman Conant and colleagues at Duke Medical School in the late 1930s. ${ }^{56}$ They endorsed the idea that there was a form of the disease specific to North America, though other forms of blastomycosis, due to other fungal species, were found on other continents. South American blastomycosis was a condition with similar symptoms, but produced 
by a different fungus - Paracoccidioides brasiliensis. Although blastomycosis typically first presented as skin lesions, there were many reports of disseminated disease, which suggested parallels with coccidioidomycosis. Doctors wondered if there was also self-limiting or sub-clinical pulmonary infection; however, there was no evidence of aerial transmission in the mode of $C$. immitis. There were few reports of blastomycosis fungus in the environment, and certainly none of concentrations likely to produce disease in humans. The mystery of the manner in which it spread led some doctors to call it the 'the enigmatic disease'. ${ }^{57}$

A study in the mid-1960s by Leo Furcolow, who was based in Kansas in the centre of an endemic area, showed that there had been only 685 confirmed cases of blastomycosis across five states between 1912 and 1964, a figure which he assumed grossly to under-estimate its actual incidence. ${ }^{58}$ Furcolow found that infection rates were higher in males than females, and there was 'a slight excess of cases among Negroes'; both factors were linked to the view that blastomycosis was associated with outdoor work or sports. A similar geographical distribution of the incidence of $B$. dermatitidis was found in dogs, so perhaps they were hosts and further credence to this link was suggested by similarities with the epidemiology of histoplasmosis. Another difficulty in mapping the disease, certainly in comparison with coccidioidomycosis, was the absence of a reliable skin test, which meant that the epidemiological picture relied on symptomatic cases.

Disseminated blastomycosis attracted the attention of clinicians because of its high mortality rate, which was typically $80 \%$, though treatment with amphotericin B brought this down to less than $20 \%{ }^{59}$ A difference with other endemic fungal diseases was that immunosuppressed patients were seemingly less likely to become infected, though if they did, severe disease was common. ${ }^{60}$ Epidemiological and clinical studies found no significant patterns in the incidence of blastomycosis, other than predisposing illness. However, it has been always been more common in men, seemingly because of outdoor exposure. Suspicions remained that its epidemiology was similar to coccidioidomycosis because the lungs were the primary site of infection, and its prevalence amongst in-migrants and construction workers. ${ }^{61}$

The low incidence of endemic blastomycosis, along with the limited systemic infection, meant that no critical mass of local specialist practitioners developed and the expertise of the few people with special knowledge was not in demand. However, scientists and clinicians in the regions affected were able to develop specialist practices by developing work on other low-level endemic mycoses, most notably histoplasmosis. 


\section{Regional mycoses III: Histoplasmosis ${ }^{62}$}

Histoplasmosis is caused by infection with the fungus Histoplasma capsulatum (H. capsulatum), and is associated with specific localities; indeed, it was sometimes referred to as Ohio Valley Fever, though it soon became clear its prevalence was wider. Its endemic areas overlapped with those of other regional fungi and researchers' work straddled different mycoses. For example, Leo Furcolow became renowned for his work on blastomycosis and histoplasmosis, and Louis Ajello combined expertise on coccidioidomycosis and histoplasmosis. ${ }^{63}$ However, unlike blastomycosis, whose low incidence meant less attention from the clinicians, histoplasmosis, similar to the medical history of coccidioidomycosis, gained prominence nationally as social changes led to an increase incidence and importance. Moreover, according to Thomas Daniel and Gerald L. Baum, a national research network emerged around the disease with work led by federal agencies. ${ }^{64}$ For Daniel and Baum, one key figure in the United States was Jan Schwarz, a Jewish émigré based in Cincinnati, Ohio, who worked on tuberculosis, before taking Conant's course at Duke and converting to mycology. From his base in Cincinnati, at the centre of the $H$. capsulatum endemicity, he became a leading national expert alongside his friend Leo Furcolow, who became known as 'Mr Histoplasmosis'.

Retrospectively, the first case of histoplasmosis has been identified in the Panama Canal Zone in 1906, when Samuel Taylor Darling reported an acute lung infection, with fever and breathlessness, caused by a protozoan that he named Histoplasma capsulatum. ${ }^{65}$ Sporadic cases were reported over succeeding decades and in 1934 its causal organism was shown to be a fungus rather than a protozoan. ${ }^{66}$ However, the disease only attracted the attention of doctors in the 1940s in relation to pulmonary tuberculosis, first in the military and then after the war in sanatoria. Recruits for the military were screened for pulmonary tuberculosis, with both a chest X-ray, which revealed active or healed lesions in the lungs, and the tuberculin skin test, which through an immune response confirmed either active or previous infection. A significant number of recruits showed, perversely, lesions in the lungs with a negative skin test. One possibility was that immunity declined over time; another was that a second disease was causing the lung lesions, and it turned out that histoplasmosis was such a disease. Fortuitously, a means to investigate the matter became available with the development of a tuberculin-type skin test for the infection, using an antigen product called histoplasmin. One study showed that of 94 men with healed lung 
lesions, 24 cases were due to tuberculosis and 70 to histoplasmosis. ${ }^{67}$ A later study by Furcolow, published in 1962, showed that histoplasmosis was responsible for lesions in $7.5 \%(3,366 / 44,882)$ of sanatorium patients from across the country, which indicated that nationally some 8,200 patients entered sanatoria with evidence of histoplasmosis, and that of these, $25 \%$ might have had active disease with tuberculosis. ${ }^{6}$

This work, facilitated by the Division of Mycotic Diseases at CDC and the US Veterans Administration, and orchestrated through a Cooperative Study Group from 1952, revealed three features of histoplasmosis. First, it showed that there were certain regions of the United States where infection with $H$. capsulatum was very common, but that the development of symptomatic disease was quite rare. It seemed that children living in endemic areas developed immunity from low-level exposure and that this gave long-term protection. Thus, those likely to develop illness were adult in-migrants without previous exposure.

Second, research on the aetiology of the infection was inconclusive for many years, but eventually yielded that the main source of infection was soil dust and that localities with accumulations of bird and bat droppings were particularly pathogenic. At times, histoplasmosis was known as 'cave disease'. Daniel and Baum describe the 'detective story' by which the aetiology was solved. Their narrative begins with a case at Camp Gruber in March 1944 when the soil as a source of the fungus was first indicated, but they show that it took several decades to determine a specific aetiology. ${ }^{69}$ Nonetheless, greater medical awareness of histoplasmosis and improved serological diagnostic testing led to more cases being identified, especially of systemic infection where, as with similar invasive mycoses, amphotericin B was the treatment of choice. ${ }^{70}$

By the 1970s, the disease had gained a higher profile, as increased population mobility around the United States brought more non-immune people to endemic areas, while anyone with active infection who moved anywhere across the country and became immuno-compromised was vulnerable to severe infection. There were also a series of epidemic outbreaks, often with small numbers affected and typically quite localised, but they were unusual, even bizarre, and attracted press and medical attention. Daniel and Baum discuss three epidemics: Mason City, Iowa in 1962 (returning in 1964) which was traced to bird rookeries; Suwanee County, Florida in 1973, linked to bats; and, the biggest of all, Indianapolis in 1978, which returned two years later. ${ }^{71}$ Final estimates for the Indianapolis outbreak were that 120,000 people were infected, with 448 persons developing clinical illness, 55 with severely disseminated disease and 19 deaths. Over the 1980s and early 1990s, histoplasmosis 
became associated in the public mind and amongst physicians with AIDS, being the commonest endemic mycosis affecting patients. ${ }^{72}$ Surveys in the early 1990 s showed that it was found in $2-5 \%$ of AIDS sufferers in endemic areas and was the first sign of infection in over half of these cases. Infection rates amongst AIDS patients as high as 25\% were found in certain cities, notably Indianapolis, Kansas City, Memphis and Nashville. Patients had quite general symptoms and treatments with the new forms of amphotericin B were successful, though maintenance of antifungals, typically the new azoles, was essential to prevent relapse.

\section{Farmer's lung and allergic bronchopulmonary aspergillosis (ABPA)}

Unlike the United States, Britain had no regionally specific mycoses linked to environmental factors of soil and climate, however, there were geographically localised conditions linked to occupation, which emerged in the 1950s. The most important was farmer's lung, which was initially linked to the Aspergilli fungi and pulmonary aspergillosis. ${ }^{73}$ Aspergillosis, the principal cause of which was Aspergillus fumigatus (A. fumigatus), was considered only at the very end of Henrici's encyclopaedic Molds, Yeasts and Actinomycetes in 1930, after coccidioidomycosis, dermatophytosis, American blastomycosis, histoplasmosis and sporotrichosis. ${ }^{74}$ However, the 15 th edition of Taylor's Practice of Medicine in 1936 presented aspergillosis as an occupational disease of handlers of birds and grain, along with those who sorted human and animal hair for various products, for example, wigs. ${ }^{75}$ Also in 1936, Richard Fawcitt, a radiologist in Ulverston, Cumberland, discussed Aspergilli spp. as the main cause of broncho-mycosis in the local farming community. ${ }^{76}$ The numbers affected were small, but this study and one by Fawcitt's colleague Munro Campbell, are seen in hindsight as the beginning of the recognition of 'farmer's lung' as an occupational disease. ${ }^{77}$ Fawcitt had also found aspergillosis amongst housecleaners, which might have been linked to new domestic technologies, such as the vacuum cleaner, which spread spore-carrying dust from exhaust vents. ${ }^{78}$

By the end of the 1930s, fungal spores had been added to pollen and house dust as causes of asthma, the prevalence of which was rising and fascinating doctors because of its complex aetiology, variable presentation, and link between the physiological and the psychological. At this time the physical basis of asthma was discussed in terms of allergic, hypersensitivity states, where the body reacted abnormally to certain substances or 'allergens' ${ }^{79}$ The largest group of allergens was plant and animal matter in the environment, with pollen and aerial 
dust understood to be the main exciting causes of allergic symptoms. Doctors debated whether the increased prevalence was due to better recognition, or because modern lifestyles made the body more vulnerable and increased exposure to allergens, or because new allergens had been created by modern farming and industry. In 1936, Grafton Brown, a Washington physician who specialised in allergy, pointed out that fungal spores were ubiquitous, present throughout the atmosphere in huge numbers and much smaller than pollen grains ${ }^{80}$ He was surprised that researchers had not considered them as allergens in house dust, especially when scientists were familiar with fungi as contaminants of their culture plates and slides in microbiology laboratories. According to Charles Thom and Margaret Church, writing in 1926, 'the Penicillia, the Aspergilli and the Mucors are the weeds of the culture room' and, of course, one now celebrated incidence of fungal contamination led to the discovery of penicillin. ${ }^{81}$ Surveys of aerial allergens increased in the 1930s, using aeroplanes to explore high altitudes and remote locations, including flights by Charles Lindberg to survey areas in northern latitudes. ${ }^{82}$ While the spores of a large number of fungal species were found to be potential allergens, they ranked well below pollen and dust. levels of known fungal disease spores found in the atmosphere were well below those of Alternaria, an ascomycete species that caused disease in plants and which emerged as main fungal suspect in causing asthma attacks. ${ }^{83}$ Next in importance were the Aspergilli.

In Britain, aspergillosis was discussed in detail in James Duncan's national survey of fungal disease in 1945, in relation to both pulmonary disease and farmer's lung. ${ }^{84}$ With pulmonary disease, cases were few and far between, and difficult to diagnose because of confusion with tuberculosis. However, Duncan was clear that 'the fungus is an essential factor in the aetiology of farmer's lung', but doubted that it was a primary inflammatory agent. ${ }^{85}$ In 1953 , Thomas Studdert, an assistant physician at the Cumberland Hospital in Carlisle, contested earlier views,

The currently quoted view that farmer's lung is an actual fungous (sic) infection of the lungs does not bear close examination. The explosive onset, spontaneous clearing, and radiological picture are totally unlike any true fungous disease, and no real evidence has been produced to support this theory. ${ }^{86}$

Studdert's alternative, still framed with the possibility of fungal involvement, was that farmer's lung was an allergic reaction 'to some material in the fungus-laden dust' ${ }^{87}$ By the mid-1950s, the number of fungi considered as allergens increased to include Penicillia, Mucors and other genera, 
yet their role was now seen as creating allergenic dust, or mechanical irritation of the bronchi and alveoli, rather than specific allergic reactions. A 1963 report by the British Industrial Injuries Advisory Council, that led to the scheduling of a variety of occupational lung conditions, linked farmer's lung to other 'dust diseases', such as thresher's lung, chaff cutter's lung and bagassosis (sugar cane handler's disease) ${ }^{88}$ It maintained the association with mouldy hay and vegetables, but said nothing about the role of specific fungi. ${ }^{89}$

A key reason why Aspergilli spp. were dropped from discussions of asthma and occupational diseases in the 1960s was the creation of the new specific condition of ABPA. ${ }^{90}$ In 1952, Kenneth Hinson, with colleagues at the London Chest Hospital, published a study of eight cases of pulmonary aspergillosis, three of which were said to be an allergic type previously unrecognised and 'caused by sensitization of the host to the fungus' ${ }^{.1}$ The condition was characterised around several symptoms: a syndrome of recurrent fevers, a changing X-ray pattern showing progressive lung damage, peripheral blood eosinophilia, and purulent sputum containing the A. fumigatus. Hinson and his colleagues were seen to have described an unusual type of aspergillosis, itself still very rare, so their claim was neither challenged nor endorsed; but it remained on the record. ABPA, as it was later styled, was not characterised by the invasive growth into tissues, rather fungi simply grew in pulmonary fluids and on the surface of lung tissues, causing inflammation.

ABPA attracted increasing attention in Britain through the 1950s and 1960s as doctors dealt with more patients with chronic lung diseases. It is again a moot point whether the decline in the incidence of pulmonary tuberculosis revealed previously submerged diseases, or whether the spectrum of disease that now faced doctors was genuinely new. Over the 1960s, there was increased incidence of lung cancer, chronic bronchitis and emphysema, which were linked to the effects of cigarettes, smoke pollution and occupation diseases. The growing incidence of non-specific chronic lung disease was captured in 1962 in the creation of the new, condition of chronic obstructive pulmonary disease (COPD). ${ }^{92}$

In both the United States and Britain there was more research on occupational diseases, which led to a greater differentiation of causes, linked to improved prevention and new regulations. ${ }^{93}$ However, ABPA was only reported in Britain and particularly in London, where the concentration of patients with chronic chest conditions allowed Jack Pepys, who was one of Britain's leading experts on allergies, to report new findings in 1959. He had investigated 145 patients who had A. fumigatus in their sputum, finding that 16 exhibited ABPA, according to Hinson's 1952 criteria. $^{94}$ Pepys published a second study in 1969, in which 
he reported on 111 patients. ${ }^{95}$ He wrote that the primary indicators of ABPA were transitory pulmonary shadows, eosinophilia of blood and sputum, evidence of allergy to A. fumigatus and fungal mycelia in sputum. Corticosteroids were the recommended therapy, having been shown to be more effective than bronchodilators and other symptomatic treatments. The condition was typically debilitating, leading to progressive deterioration in lung function, though in some patients the condition 'burnt out' and they 'recovered'.

American allergists and pulmonary specialists were curious about why ABPA was not found across the Atlantic. In 1969, Raymond Slavin and colleagues at the St. Louis University School asked:

Why is [allergic] aspergillosis such a rarity in the United States? It would seem that the climate and geography of England does not make a profound difference since $A$. fumigatus is commonly reported in air sampling surveys in this country. In addition, as stated previously, secondary aspergillosis is not uncommon. It appears then that a failure of recognition and errors of omission account for the rarity of allergic aspergillosis in the United States... With the proper appreciation of the characteristics of allergic aspergillosis, both laboratory and clinical, this disease may be more frequently recognized and take its place with such hypersensitivity pneumonitides as pigeon breeders' disease, bagassosis and farmers' lung. ${ }^{96}$

As late as 1977, APBA was being discussed as an 'emerging disease' in the United States, due 'to increased awareness by physicians, increased referral, better diagnostic modalities, and earlier bronchography'. ${ }^{97}$ Studies in the 1970s comparing the incidence of APBA in London and Cleveland showed similar levels of sensitivity to A. fumigatus antigens in asthmatics in both cities ( $23 \%$ and $28 \%$, respectively), with the differences in prevalence attributed to exposure. ${ }^{98}$ However, the incidence of the condition was on the rise in both countries, contributing to the overall increase in asthma, which has been widely discussed and attributed to many factors, from greater awareness to modern lifestyles. ABPA was soon recognised in most countries as the most common form of fungal-induced allergic lung disease and, though a largely chronic condition, was known to produce acute episodes. ${ }^{99}$

By the early 1980s, ABPA was a disease defined by eight diagnostic criteria, which were: asthma, an immediate positive skin test to Aspergillus antigens; presence of antibodies for A. fumigatus; elevated total serum immunoglobulin E (IgE); bronchial damage revealed by X-ray; high levels of white blood cells; proximal dilatation of the bronchi and elevated 
serum immunoglobulin levels of IgE-Af and lgG-Af compared with mould-sensitive asthmatic patients. ${ }^{100}$ This demanding series, which combined clinical, X-ray, laboratory and functional criteria, meant that differential diagnosis against other lung conditions, such as pneumonia, bronchiectasis and carcinoma, was difficult in practice. There were problems about standardisation between individual clinicians, let alone across clinics and countries. Treatment was largely symptomatic, mostly with prednisone, an anti-inflammatory corticosteroid; however, some doctors tried antifungal antibiotics, given by inhalation and as well as systemically. ${ }^{101}$ The chronic character of ABPA led to investigations into the degree of the destruction of lung tissue in severe cases, recognition of which led eventually to the designation, by David Denning and his colleagues in Manchester, of a group of patients with the new condition of severe asthma associated with fungal sensitivity (SAFS). ${ }^{102}$

By the early 1990s, ABPA was mainly identified with two groups: chronic asthma sufferers and people with cystic fibrosis. In both groups, inflamed lung tissues, accumulated exudates, dilated bronchi, impaired breathing and other factors created the conditions for Aspergilli to grow and prompt an allergic response. Epidemiological studies suggested that $1-5 \%$ of asthma sufferers were affected by ABPA, which was, of course, a fast growing number of individuals. In 1991, the American Academy of Allergy, Asthma and Immunology (AAAAI) formed an ABPA Committee, which attempted to determine the incidence of the condition. Initial results suggested ABPA affected less than 1\% of asthma patients, though without a standard diagnosis and poor reporting the figure was speculative. ${ }^{103}$

A higher incidence of ABPA was found amongst people with cystic fibrosis, which was a rapidly growing group due to the increase in life expectancy because of improved management of the condition. ${ }^{104}$ Studies at the end of the 1970s showed that around 10\% of children attending the cystic fibrosis clinics had the symptoms of ABPA. ${ }^{105}$ However, fungal allergy was just one of a number of lung infections this group was vulnerable to and it was far less prevalent than those caused by bacteria and viruses. ${ }^{106}$ A study published in 1990 reported that the main pathogens affecting people with cystic fibrosis were Pseudomonas aeruginosa (60\%) and Staphylococcus aureus (27\%), with ABPA in the range of $0.5-11 \% .{ }^{107}$ However, it was more common in older children and adults, at around 25\%; hence, its importance was likely to grow. ${ }^{108}$

In 2001, a report by staff at the CDC in Atlanta presented an overview of mortality from invasive mycoses in the United States from 1980 to 
1997. ${ }^{109}$ The data they collected showed that 'deaths in which an infectious disease was the underlying cause, those due to mycoses increased from the tenth most common in 1980 to the seventh most common in 1997', with the annual number of deaths increasing from 1,557 to 6,534 . They confirmed that there had been 'a marked upward trend in overall mortality due to the invasive mycoses', and highlighted the growing importance of immuno-compromising conditions, particularly HIV/AIDS. ${ }^{110}$ What was interesting was that the regionally specific mycoses discussed in this chapter only registered in the summary when they affected patients with HIV/AIDS, indeed, the disease was 'a major determinant of the trend in overall mortality from histoplasmosis'. ${ }^{111}$ However, the report supported the view that social changes were major factors in the fluctuating incidence of fungal disease, as with coccidioidomycosis in Arizona, where the increase was due to 'an influx into the state of older nonimmune individuals who were susceptible to acute infection and more likely to manifest symptomatic illness'. ${ }^{112}$ In other places, it was not so much the arrival of virgin human soil, but the wider and more intense circulation of the 'seeds' of infection, literally thrown up by construction and extreme climate events.

In discussing overall mortality, the CDC report confirmed what doctors' experience had told them:

The two major factors responsible for the emergence of fungal infections have been the HIV disease epidemic and the many advances of modern medicine (including solid organ and bone marrow transplantation) that enable or prolong the survival of critically ill and susceptible patients. In addition, the aging of the population has increased the number of susceptible persons. ${ }^{113}$

To which should be added greater medical awareness, plus new and more sensitive diagnostic technologies. However, the report showed that antifungal drugs had reduced mortality in certain groups and from certain infections; the main exception was aspergillosis, the mycosis most associated with 'advances in modern medicine', which we move on to in the next chapter.

Except where otherwise noted, this work is licensed under a Creative Commons Attribution 3.0 Unported License. To view a copy of this license, visit http://creativecommons.org/licenses/by/3.0/ 